\title{
Primary Hepatic Follicular Lymphoma : A Case Report and Discussion of Chemotherapy and Favorable Outcomes
}

\author{
Hiroshi Gomyo ${ }^{1)}$, Yoshitoyo Kagami ${ }^{1)}$, Harumi Kato ${ }^{1)}$, Takakazu Kawase ${ }^{1)}$, Aya Ohshiro ${ }^{1)}$, \\ Takashi Oyama $^{2}$, Yoshikazu Kamiya ${ }^{2}$, Hirofumi Taji ${ }^{1}$, Shigeo Nakamura ${ }^{3)}$, Michinori Ogura ${ }^{1)}$, \\ and Yasuo Morishima ${ }^{1)}$
}

This report concerns a rare case of follicular lymphoma with features suggestive of primary hepatic lymphoma. At the disease onset, multiple nodular lesions in the liver and small para-aortic nodes were detected by abdominal magnetic resonance imaging without generalized lymphadenopathy. After careful observation for three months, lymphadenopathy was observed in the right occipital, para-aortic, and bilateral inguinal regions. The patient was treated with R-CHOP (rituximab, cyclophosphamide, doxorubicin, vincristine and prednisone) and achieved complete remission for more than 2 years. To the best of our knowledge, this is the ninth report of primary hepatic follicular lymphoma. Insufficient cases have been reported to determine the long-term outcome and clinical characteristics of primary hepatic follicular lymphoma. However, it seems that patients with primary hepatic follicular lymphoma that are treated with appropriate chemotherapy with or without surgical resection have favorable outcomes.〔J Clin Exp Hematopathol 47(2) : 73- 77, 2007〕

Keywords: primary hepatic lymphoma, primary hepatic follicular lymphoma, R-CHOP

\section{INTRODUCTION}

Primary hepatic lymphoma is a rare disease, representing $0.4 \%$ of extranodal non-Hodgkin's lymphomas. ${ }^{1}$ The most common histological type is reported to be diffuse large Bcell lymphoma. ${ }^{2}$ Follicular lymphoma of the liver is extremely rare and accounts for only $1-4.4 \%$ of primary hepatic lymphomas. ${ }^{2-4}$ Because of its rarity, the clinical features of primary hepatic follicular lymphoma have not been fully identified. In this case report, we discuss the clinical characteristics of primary hepatic follicular lymphoma and the potential relationship between chemotherapy and favorable outcomes.

\section{CASE REPORT}

A 65-year-old Japanese woman presented to a local hospital in October 2003 complaining of right upper abdominal

\footnotetext{
Received : Jun 15, 2006

Revised : Feb 27, 2007

Accepted: Mar 25, 2007

1) Department of Hematology and Cell Therapy, Aichi Cancer Center, Nagoya, Japan

${ }^{2)}$ Department of Medical Oncology, Aichi Cancer Center, Nagoya, Japan

${ }^{3}$ Department of Pathology and Molecular Diagnostics, Aichi Cancer Center, Nagoya, Japan

Address correspondence and reprint request to Yoshitoyo Kagami, Aichi Cancer Center, Kanokoden 1-1, Chikusaku, Nagoya, Aichi 464-8681, Japan

E-mail : ykagami@aichi-cc.jp
}

and lower back pain. She had a history significant for acute cholecystitis at the age of 33. A physical examination revealed no abnormal findings except for right costovertebral angle tenderness. There was no palpable superficial lymphadenopathy. The liver and spleen were normal in size, and there was no history of fever, night sweats, or weight loss. Laboratory test results were: hemoglobin $12.6 \mathrm{~g} / \mathrm{dl}$, white blood cells $5000 / \mu \mathrm{l}$ with normal differential count, platelet count $20.1 \times 10^{4} / \mu 1$. Aspartate aminotransferase and alanine aminotransferase were within normal limits. However, alkaline phosphatase (272 U/1 ; normal 90 - 238) and lactate dehydrogenase (LDH) levels (487 U/1; normal 228-432, LDH isozyme was not analyzed) were slightly elevated. Serum $a^{-}$ fetoprotein and carcinoembryonic antigen levels were normal. Serology was negative for hepatitis B surface antigen, hepatitis B surface antibody, hepatitis C virus (HCV), and human immunodeficiency virus. Computed tomography (CT) of the abdomen showed multiple hypodense lesions in both lobes of the liver with no evidence of splenic involvement. There was no sign of lymphadenopathy except for small para-aortic nodes that measured $1.0 \mathrm{~cm}$ along their major axes. Chest $\mathrm{X}$ ray and upper gastrointestinal endoscopic findings were normal. This patient was then referred to another hospital for closer examination of the liver masses detected by CT. Subsequent magnetic resonance imaging revealed multiple lesions that were hypointense on T1-weighted imaging and 
hyperintense on T2-weighted imaging (Fig. 1). A hepatic angiogram performed on December 16th 2003 showed no neovascularity or tumor stain. Because a metastastic malignant hepatic tumor was suspected, epirubicin was infused into the hepatic artery and on December 18th, a needle biopsy of the tumor under ultrasound guidance was performed.

Histological analysis was performed to determine the diagnosis and showed diffuse infiltration of small- to medium-sized cells with slightly angulated or twisted nuclei, a feature similar to that of follicular lymphoma (Fig. 2). Immunostaining of the tumor cells showed reactivity for CD10, CD20 but not for CD3. Based on these findings, the liver tumor was diagnosed as an infiltration of follicular lymphoma cells.

After careful observation for three months, the patient was referred to our hospital for treatment. At that time, lymphadenopathy was observed in the right occipital $(2.0 \mathrm{x}$ $1.0 \mathrm{~cm})$ and bilateral inguinal regions $(1.5 \mathrm{x} 1.5 \mathrm{~cm}$ to $1.5 \mathrm{x}$ $2.0 \mathrm{~cm}$ ), but there was no involvement of the bone marrow or gastrointestinal tract. As the lymphadenopathy had developed over a short period of time, a right inguinal lymph node biopsy was performed to exclude histological transformation. Flow cytometry identified the lymphoma cells as CD10, CD19, and CD20 positive exhibiting $\lambda$ light chain restriction. The sampled cells were CD5 and CD23 negative. Chromosomal analysis of 7 lymphoma cells identified 5 of them as $46, X X, \operatorname{del}(10)(\mathrm{q} ?)), \mathrm{t}(14 ; 18)(\mathrm{q} 32 ; \mathrm{q} 21)$. Immunohistochemistry further showed the neoplastic cells to be positive for bcl-2 and CD20. The resultant pathologic diagnosis was follicular lymphoma, grade 1 . The patient was then treated with 6 courses of R-CHOP (rituximab, cyclophosphamide, doxorubicin, vincristine, and prednisone),

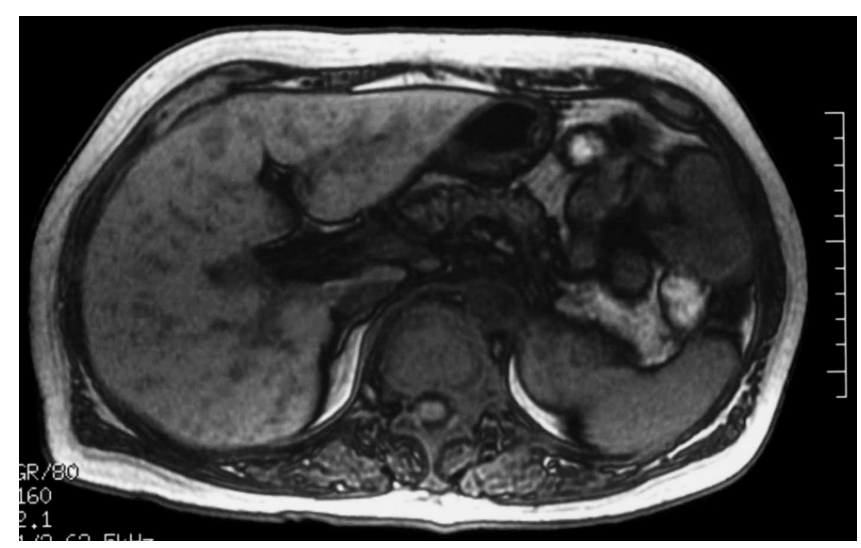

Fig. 1. Magnetic resonance imaging: T1-weighted image of the liver demonstrates multiple hypointense lesions. achieved complete remission, and for greater than 2 years remained in stable health without additional therapy.

\section{DISCUSSION}

Whereas secondary involvement of the liver by nonHodgkin's lymphoma is relatively common, involving the liver in as many as $21 \%$ of advanced non-Hodgkin's lymphoma cases, primary hepatic lymphoma is extremely rare. ${ }^{8}$ The most common histological type of primary hepatic lymphoma is diffuse large B-cell lymphoma (DLBCL), which accounts for $46-95.8 \%$ of cases. ${ }^{2}$ Primary hepatic follicular lymphoma is even rarer, accounting for only $1-4.4 \%$ of primary hepatic lymphoma. ${ }^{2-4}$ To the best of our knowledge, ours is the ninth reported case of primary hepatic follicular lymphoma.

The diagnostic criteria for primary hepatic lymphoma are not well defined. Caccamo et al. ${ }^{5}$ proposed strict diagnostic criteria requiring confirmation of the liver as the primary site. On the other hand, Lei ${ }^{2}$ proposed the following criteria for the diagnosis of primary hepatic lymphoma: (1) the symptoms are mainly caused by liver involvement at presentation; (2) distant lymphadenopathy is neither palpable clinically at presentation nor detected during radiological staging studies; and (3) no leukemic blood picture is observed on the peripheral blood film. Others have accepted cases with extrahepatic involvement at the time of diagnosis as being "primary" if extension from the liver was considered to be the most likely cause of the secondary site of involvement. ${ }^{6,7}$ We could not identify the primary site in our patient with absolute certainty due to the existence of small para-aortic nodes detected by abdominal MRI at the time of initial presentation. Nevertheless, the diagnosis of primary hepatic lymphoma is supported by the striking hepatic involvement seen in this patient.

Further, this diagnosis was supported by histologic and marker studies of the biopsy sample. Because of the limited size of the needle biopsy specimen, the full follicular pattern was hardly observable. Yet, the cytomorphologic features of our case were in keeping with those of prototypic follicular lymphoma, i.e., a cleaved/non-cleaved cellular pattern. The positive immunoreactivity results for CD10, CD20, and BCL2 of the tumor cells provided additional evidence in support of the follicular lymphoma diagnosis.

The following is a summary of the clinical findings of the 9 reported cases (Table 1). The age of the patients at the time of diagnosis ranged from 47 years to 82 years (median age 63 years), so that middle-aged and elderly individuals predominated. The median age for primary hepatic follicular lymphoma was slightly higher than that for the overall population of follicular lymphoma (59 years), ${ }^{9}$ and also higher than the 53 years $^{2}$ and 56 years $^{3}$ age reported in recent reviews including that for various histological subtypes of lymphoma. 
$2 A$

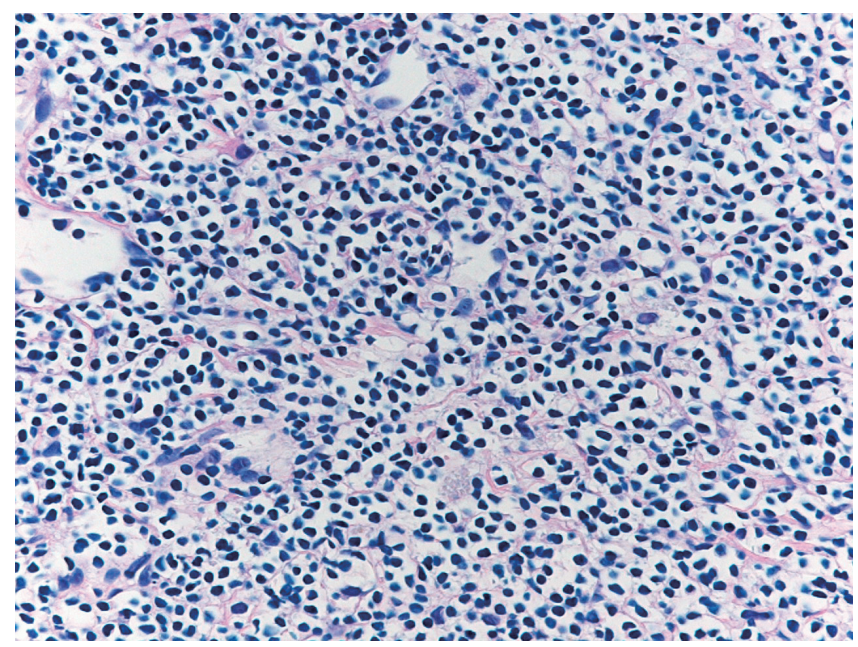

$2 C$

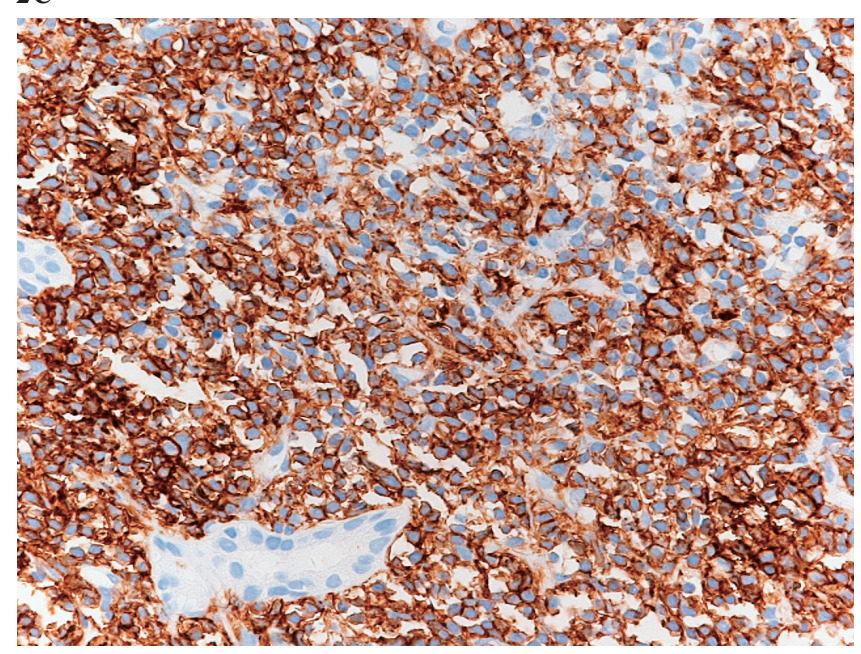

$2 B$

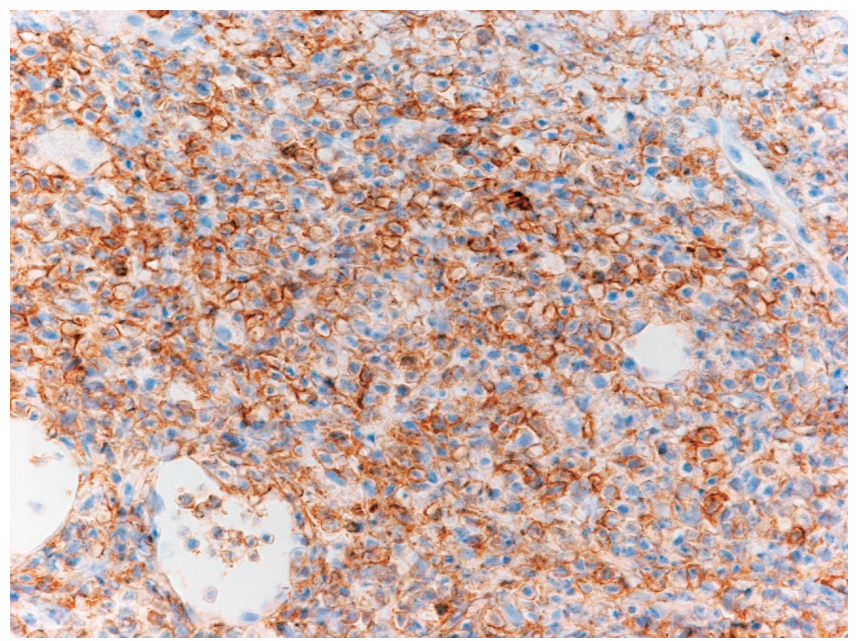

$2 D$

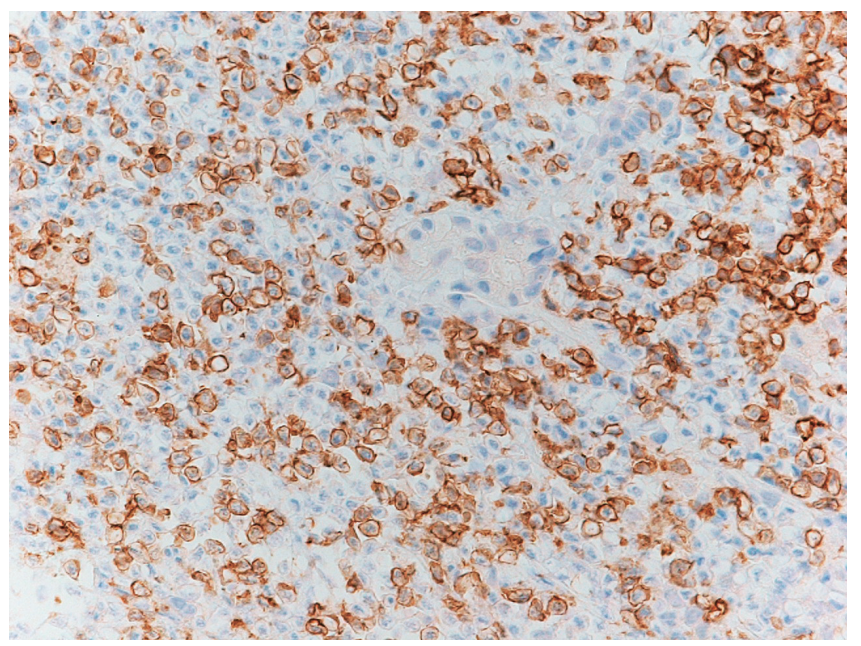

Fig. 2. Histological and immunohistochemical photographs of liver tumor. (2A) Hematoxylin and eosin staining, x200; (2B) : antiCD10 staining, x200; (2C) : anti-CD20 staining, x200; (2D): anti-CD3 staining, x200. Photographs show diffuse infiltration of small- to medium-sized cells which are positive for CD10 and CD20, and negative for CD3.

The male-to-female ratio was $2: 1$, which is consistent with other studies ${ }^{2,3}$ demonstrating male predominance. On the contrary, general follicular lymphoma has a slight female predominance $(1: 1.4){ }^{9} \quad$ The most frequently presented symptoms were upper abdominal pain or discomfort, although 5 of the 9 patients $(55.5 \%)$ were asymptomatic., ${ }^{2,3}$ The most frequent histological type was follicular lymphoma, grade 1 ( 7 of 9 cases). Four of the 9 cases had more than two lesions in the liver. Lastly, serological evaluations of hepatitis $\mathrm{C}$ virus antibody and hepatitis B surface antigen were obtained for 7 patients and showed that the prevalence for both was $28.6 \%$ ( 2 of 7 cases).

The rate of HCV and hepatitis B virus (HBV) infection is not necessarily increased in primary hepatic follicular lymphoma compared to that of general non-Hodgkin's lymphoma (NHL), and further study of the exact pathogenic role of

\section{$\mathrm{HCV}$ and $\mathrm{HBV}$ is needed.}

Page et al. ${ }^{10}$ reported that 6 of $10(60 \%)$ patients with primary hepatic lymphoma ( $96 \%$ of patients were DLBCL) tested positive for $\mathrm{HCV}$, whereas 2 of $15(13 \%)$ patients tested positive for HBV ; these two patients were also positive for HCV. Therefore, authors of this study speculated that $\mathrm{HCV}$ plays some role in the pathogenesis of neoplasm. ${ }^{10}$ Moreover, Ohsawa et al. ${ }^{4}$ reported the occurrence of chronic liver diseases before the onset of primary hepatic lymphoma in $7(44 \%)$ of 16 Japanese patients and $5(9.6 \%)$ of 52 Western patients. They concluded that chronic liver diseases including HBV induced hepatitis might play an important role in the development of hepatic lymphoma, especially in Japanese patients. On the other hand, Lei $^{2}$ reported in a literature review that preexisting liver disease including liver cirrhosis caused by HBV or HCV had an incidence of less 
Table 1. Summary of the clinical findings of the 9 reported cases of primary hepatic follicular lymphoma

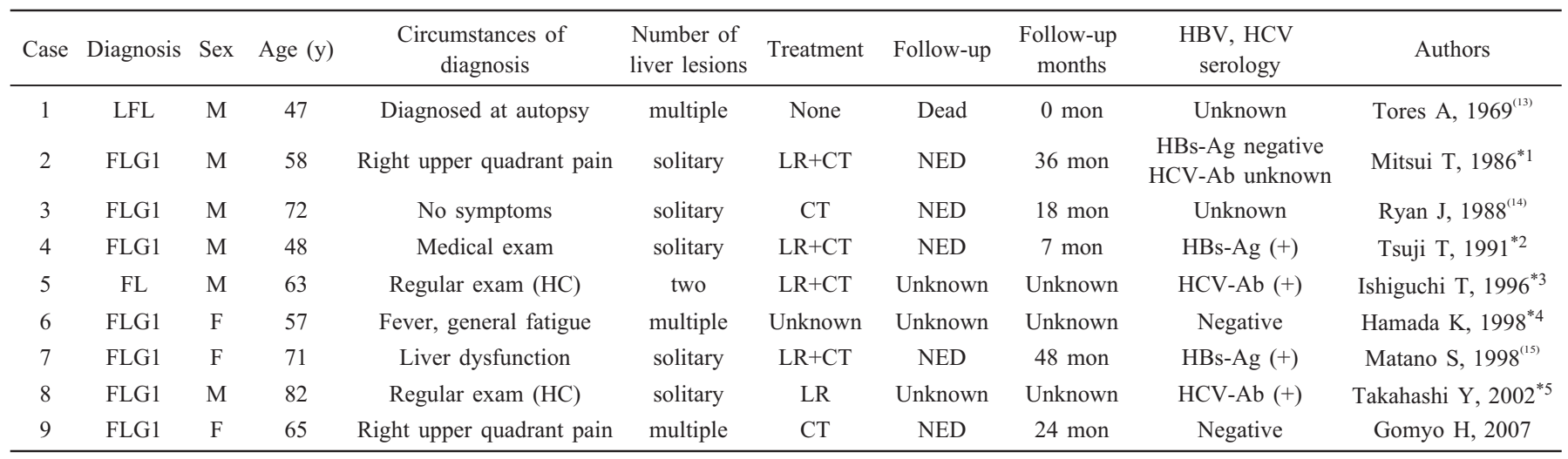

LFL : lymphocytic follicular lymphoma; FLG1 : follicular lymphoma grade 1; HC : hepatitis C ; LR : liver resection ; CT : chemotherapy ; NED : no evidence of disease : ${ }^{* 1}$ Geka $48: 745-748,1986:{ }^{* 2}$ Hukubu-Gazoushindan 11: 631-639, $1991:{ }^{* 3}$ Wakayama-Igaku $47: 397,1996:{ }^{* 4}$ Okinawa-Igakkai-Zassi 37 : 56- 58, $1998:{ }^{* 5}$ Shindan-Byori 19: 126- 130, 2002

than $9 \%$ in the setting of primary hepatic lymphoma. He concluded that there is insufficient evidence to support HBV or $\mathrm{HCV}$ as etiologic factors. In general, $\mathrm{HCV}$ infection rates in B-cell lymphoproliferative disorders are $10-48 \%,{ }^{11}$ and HBV infection rates in NHL are 3.2-29.5\%. ${ }^{12}$

Primary hepatic lymphoma traditionally is considered to be an aggressive disease with a poor prognosis. ${ }^{2}$ Of the 9 cases considered here, information about the treatment and follow-up of 5 patients was available. Two patients were treated with therapeutic surgical resection followed by adjuvant chemotherapy, one patient underwent therapeutic surgical resection alone and received chemotherapy for relapse, and 2 patients (including our case) were treated with chemotherapy alone. All but one patient were treated with CHOP-like chemotherapy. The median survival of these 5 cases was 18 mon, and all of them remained alive without disease progression. Although the follow-up period is relatively short, this finding suggests that patients with primary hepatic follicular lymphoma have a favorable outcome if they receive appropriate chemotherapy with or without surgical resection. Further investigations are needed to clarify the clinical characteristics of this rare disease.

\section{REFERENCES}

1 Freeman C, Berg JW, Cutler SJ : Occurrence and prognosis of extranodal lymphomas. Cancer 29: 252-260, 1972

2 Lei KI: Primary non-Hodgkin's lymphoma of the liver. Leuk Lymphoma 29: 293-299, 1998

3 Bronowicki JP, Bineau C, Feugier P, Hermine O, Brousse N, Oberti F, Rousselet MC, Dharancy S, Gaulard P, Flejou JF, Cazals-Hatem D, Labouyrie E : Primary lymphoma of the liver : clinical-pathological features and relationship with HCV infection in French patients. Hepatology 37 : 781- 787, 2003

4 Ohsawa M, Aozasa K, Horiuchi K, Kataoka M, Hida J, Shimada
H, Oka K, Wakata Y : Malignant lymphoma of the liver. Report of five cases and review of the literature. Dig Dis Sci 37 : 1105-1109, 1992

5 Caccamo D, Pervez NK, Marchevsky A : Primary lymphoma of the liver in the acquired immunodeficiency syndrome. Arch Pathol Lab Med 110 : 553-555, 1986

6 Aghai E, Quitt M, Lurie M, Antal S, Cohen L, Bitterman H, Froom P : Primary hepatic lymphoma presenting as symptomatic immune thrombocytopenic purpura. Cancer 60 : 2308-2311, 1987

7 Sutton E, Malatjalian D, Hayne OA, Hanly JG : Liver lymphoma in systemic lupus erythematosus. J Rheumatol 16 : 1584-1588, 1989

8 Chabner BA, Johnson RE, Young RC, Canellos GP, Hubbard SP, Johnson SK, DeVita VT Jr: Sequential nonsurgical and surgical staging of non-Hodgkin's lymphoma. Ann Intern Med 85 : 149- 154, 1976

9 The Non-Hodgkin's Lymphoma Classification Project: A clinical evaluation of the International Lymphoma Study Group classification of non-Hodgkin's lymphoma. Blood 89 : 3909-3918, 1997

10 Page RD, Romaguera JE, Osborne B, Medeiros LJ, Rodriguez J, North L, Sanz-Rodriguez C, Cabanillas F : Primary hepatic lymphoma: favorable outcome after combination chemotherapy. Cancer 92: 2023-2029, 2001

11 Turner NC, Dusheiko G, Jones A : Hepatitis C and B-cell lymphoma. Ann Oncol 14: 1341-1345, 2003

12 Kim JH, Bang YJ, Park BJ, Yoo T, Kim CW, Kim TY, Heo DS, Lee HS, Kim NK: Hepatitis B virus infection and B-cell nonHodgkin's lymphoma in a hepatitis B endemic area : a casecontrol study. Jpn J Cancer Res 93 : 471-477, 2002

13 Torres A : Primary lymphocytic follicular lymphoma of liver. Cancer 23 : 1185-1199, 1969

14 Ryan J, Straus DJ, Lange C, Filippa DA, Botet JF, Sanders LM, Shiu MH, Fortner JG : Primary lymphoma of the liver. Cancer $61: 370-375,1988$

15 Matano S, Nakamura S, Annen Y, Hattori N, Kiyohara K, Kakuta 
Primary hepatic follicular lymphoma

K, Kyoda K, Sugimoto T : Primary hepatic lymphoma in a patient with chronic hepatitis B. Am J Gastroenterol 93 : 2301-2302, 1998 